\title{
CARACTERISATION DES LOIS FORTEMENT UNIMODALES
}

\author{
P. CAPÉRAÀ, ${ }^{*}$ Université Laval
}

STRONG UNIMODALITY; DISPERSIVE ORDERING; TAIL ORDERING

Droste et Wefelmeyer (1985) caractérisent la forte unimodalité d'une loi $P$ par

$$
\begin{aligned}
& P \text { fortement unimodale } \Leftrightarrow P * Q \text { plus dispersée que } \\
& P \text { pour toute loi } Q \text {. }
\end{aligned}
$$

Une des définitions d'une loi $R$ plus dispersée qu'une loi $P(R>P)$ est donnée par Bickel et Lehmann (1979):

$$
R \underset{d}{R>P \Leftrightarrow H^{-1}(u)-F^{-1}(u) \text { croissant pour } u \in(0,1)}
$$

$F$ et $H$ étant les fonctions de répartition de $P$ et $R$ respectivement et $F^{-1}(u)=$ $\inf _{x}\{x ; F(x) \geqq u\}$ (même chose pour $H$ ). Droste et Wefelmeyer font l'hypothèse que la loi $P$ admet une densité $f$ partout strictement positive, donc dans ce cas là les fonctions réciproques $F^{-1}$ et $H^{-1}$ existent.

Le but de cette lettre est de signaler que le résultat démontré par ces deux auteurs est une conséquence immédiate d'un théorème démontré par Pratt (1964). En effet dans cet article, Pratt considère une fonction $F$ réelle (avec les notations de Droste et Wefelmeyer) possédant une dérivée $f$ partout positive. Définissons la fonction $c(\cdot ; Q)$ par

$$
F(x+c(x ; Q))=\int F(x-y) d G(y)=H_{Q}(x)
$$

où $G$ est la fonction de répartition de la loi $Q$. Alors puisque $F$ est strictement croissante, on a, pour tout réel $x$,

$$
c(x ; Q)=-x+\left(F^{-1} \circ H_{Q}\right)(x) \text {. }
$$

Pratt démontre (adaptation de $(\mathrm{a})$ et $(\mathrm{b})$ des théorèmes 1 et 2 )

$$
\text { pour toute loi } Q, c(\cdot ; Q) \text { décroissant } \Leftrightarrow
$$

$$
\text { pour tout réel positif } z, f(\cdot) / f(\cdot+z) \text { croissant. }
$$

On voit alors aisément que

$$
\text { (2) } \Leftrightarrow \text { pour toute loi } Q, H_{Q}^{-1}-F^{-1} \text { croissant sur }(0,1)
$$

et

$$
\text { (3) } \Leftrightarrow P \text { fortement unimodale, }
$$

d'où le résultat (1) démontré par Droste et Wefelmeyer.

Received 15 November 1985.

* Postal address: Départment de mathématiques, statistique et actuariat, Université Laval, Québec, PQ, Canada G1K 7P4. 
Du théorème de Pratt ou de Droste et Wefelmeyer, on déduit et complète le résultat annoncé par Bickel et Lehmann (1975) concernant les lois contaminées. En effet, soit $P$ une loi symétrique par rapport à zéro, de fonctions de répartition $F$ admettant une densité $f$ partout strictement positive et considérons $R_{Q}$ une loi résultant de la contamination de $P$ par une loi $Q$ définie sur $\mathbb{R}^{+}$. En notant $G$ et $H_{Q}$ les fonctions de répartition de $Q$ et $R_{Q}$ respectivement on a

$$
H_{Q}(x)=\int_{0}^{+\infty} F(x y) d G(y) .
$$

Considérons encore l'ordre de Lawrence (1975) défini par

$$
\underset{r}{P} R_{Q} \Leftrightarrow H_{Q}^{-1} / F^{-1} \text { croissant sur }\left(\frac{1}{2}, 1\right)
$$

et qui permet de comparer deux lois suivant le poids de leurs queues. Alors on déduit de (1)

(4) pour toute loi $Q, P<R_{Q} \Leftrightarrow P$ est à rapport de vraisemblance monotone pour un paramètre d'échelle.

En effet si $X$ est une variable aléatoire de loi $P$ à rapport de vraisemblance monotone pour un paramètre d'échelle, alors $\log |X|$ a une loi fortement unimodale et le résultat (4) se montre aisément en remarquant (Shaked (1982))

$$
\underset{r}{P} R_{Q} \Leftrightarrow \operatorname{loi} \text { de } \log |X|{ }_{d} \text { loi de } \log |Y|,
$$

où $Y$ a pour loi $R_{Q}$.

\section{Bibliographie}

Bickel, P. J. AND LehMANN, E. L. (1975) Descriptive statistics for nonparametric models II. Location. Ann. Statist. 3, 1045-1069.

Bickel, P. J. AND LEHMANN, E. L. (1979) Descriptive statistics for nonparametric models IV. Spread. In Contributions to Statistics, Jaroslav Hajek Memorial Volume, ed. J. Jureckova, Riedel, Dordrecht, 33-40.

Droste, W. AND WeFELMEYER, W. (1985) A note on strong unimodality and dispersivity. $J$. Appl. Prob. 22, 235-239.

LaWrence, M. J. (1975) Inequalities of $s$-ordered distributions. Ann. Statist. 3, 413-428.

Pratt, J. W. (1964) Risk aversion in the small and in the large. Econometrica 32, 122-136.

SHAKED, M. (1982) Dispersive ordering of distributions. J. Appl. Prob. 17, 310-320. 